\section{ORIGINAL RESEARCH}

O. Naggara

T.E. Darsaut

I. Salazkin

G. Soulez

F. Guilbert

D. Roy

A. Weill

G. Gevry

J. Raymond

\title{
A New Canine Carotid Artery Bifurcation Aneurysm Model for the Evaluation of Neurovascular Devices
}

\begin{abstract}
BACKGROUND AND PURPOSE: Stents are increasingly used for coiling of difficult aneurysms, to reduce the risk of recurrences, or to modify blood flow. Currently available bifurcation aneurysm models are ill-suited to assess stent performance before clinical use. We designed a new wide-neck canine T-type bifurcation aneurysm model. Its potential value as a training tool as well as in the evaluation of new techniques or embolic agents was assessed. Our first task was to verify that recurrences occurred after satisfactory coiling. A second aim of this preliminary work was to assess if the new model could recreate the technical challenges involved in bifurcation aneurysms.
\end{abstract}

\begin{abstract}
MATERIALS AND METHODS: We introduce a new canine wide-neck bifurcation aneurysm model, created by using a vein pouch at the apex of an end-to-side anastomosis of the carotid arteries, with flow reversal in the proximal RCA by ligation of the innominate artery. Three aneurysms were treated with coil embolization, 10 were treated with stents (7 self-expandable, 3 balloon-expandable), and 3 were left untreated. Aneurysms were followed by duplex ultrasonography and angiography, and studied with macroscopic photography after euthanasia $11.8 \pm 3.9$ months after surgery.
\end{abstract}

RESULTS: All aneurysms remained patent at $9.0 \pm 3.6$ months' follow-up. Coiling led to recurrences by 3 months in all 3 cases. Stent placement was technically difficult in all cases and did not lead to aneurysm thrombosis or neointimal closure of the aneurysm neck at 3 months.

ConcLusions: This model may be suitable for studying the effects of endovascular treatment on aneurysm and branch occlusion rates, for preclinical testing of stents and other intravascular devices, and for training students of endovascular technique.

ABBREVIATIONS: IT = innominate trunk; $\mathrm{LCA}=$ left carotid artery; $\mathrm{RCA}=$ right carotid artery; $\mathrm{SCA}=$ subclavian artery; $\mathrm{VP}=$ venous pouch

C oil embolization of ruptured intracranial aneurysms leads to better clinical outcomes than surgical clipping. ${ }^{1}$ The endovascular approach is less definitive than surgical clipping, however, with a higher incidence of residual and recurrent aneurysms at follow-up imaging. ${ }^{2}$ New techniques or materials designed to improve long-term results of endovascular treatment must be evaluated in experimental models that reproduce the problem investigators want to address before they can be proposed for clinical application. ${ }^{3}$ Currently available aneurysm models are not sufficiently challenging to assess preclinical in vivo stent performance. ${ }^{4}$ We designed a new wideneck canine T-type bifurcation aneurysm model. Its potential value as a training tool as well as in the evaluation of new techniques or embolic agents was assessed. The model combines a right carotid lateral wall aneurysm, on the opposite side of a $90^{\circ}$ end-to-side anastomosis between the left and right carotid arteries, and reversal of flow in the proximal RCA by the steal phenomenon created by clipping of the innominate

\section{Received July 23, 2009; accepted after revision September 27}

From the Interventional Neuroradiology Research Unit, Department of Radiology, Centre Hospitalier de I'Université de Montréal, Notre-Dame Hospital, Montreal, Canada.

This study was funded by the Canadian Institutes of Health Research and the Heart and Stroke Foundation of Québec.

Please address correspondence to Jean Raymond, MD, CHUM-Notre-Dame Hospital, Interventional Neuroradiology Research Lab (NRI), 1560 Sherbrooke East, Pavilion Simard, Suite Z12909, Montreal, OC H2L 4M1, Canada; e-mail: jean.raymond@umontreal.ca

\section{Indicates open access to non-subscribers at www.ajnr.org}

DOI 10.3174/ajnr.A1929 artery. Our first task was to verify that recurrences occurred after satisfactory coiling. A second aim of this preliminary work was to assess if the new model could recreate the technical challenges involved in bifurcation aneurysms.

\section{Materials and Methods}

\section{Aneurysm Construction}

Protocols for animal experimentation were approved by the Institutional Animal Care Committee in accordance with guidelines of the Canadian Council on Animal Care. All procedures were performed in 7- to 10-kg male beagles under general anesthesia. Large, wide-neck aneurysms $(n=16)$ were constructed after a T-type bifurcation was created between the 2 common carotid arteries, with subsequent flow reversal from clipping the ipsilateral innominate artery (Fig 1). The details of the surgical procedure are as follows: Through a linear vertical midline incision between the larynx and sternum, the accessible segment of the left external jugular vein is harvested, inverted to remove potential valvular obstructions, and then stored in heparinized saline at room temperature until needed for vein pouch creation. The pretracheal fascia is divided in the midline to gain access to both common carotid arteries. The left common carotid is mobilized from the inferior portion of the wound to the origin of the thyroid artery. A temporary clip is placed proximally on the LCA, which is then ligated and divided distally. The left carotid is then tunneled behind the esophagus and made to adopt a natural lie where it meets the opposing right carotid at a $90^{\circ}$ angle.

On the right side, the carotid dissection is carried inferiorly into the mediastinum to expose the innominate artery, which is then oc- 


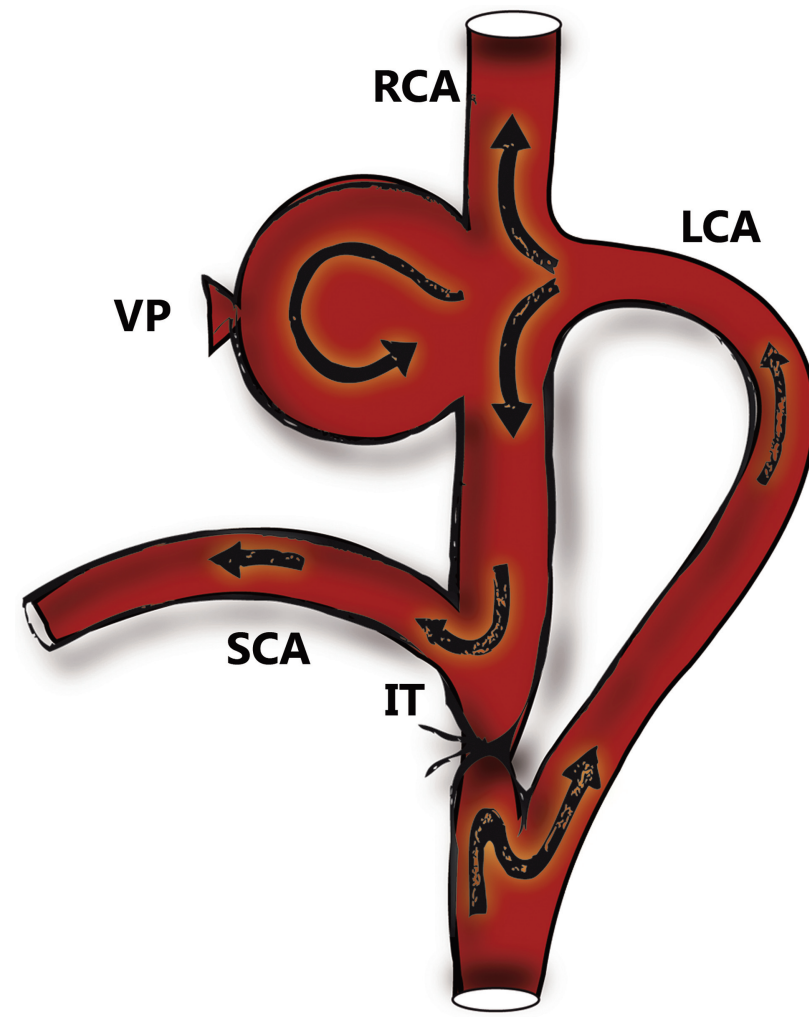

Fig 1. Surgical construction of novel carotid bifurcation aneurysm model. Note the flow reversal in the proximal $\mathrm{RCA}$.

cluded by a permanent vascular clip placed below the origins of the left carotid and the right subclavian arteries, taking care to avoid the recurrent laryngeal and vagus nerves. Temporary clips are then applied to the segment of RCA and a 5-mm arteriotomy is created on the medial surface opposite the transected LCA ostium. After fishmouthing the distal left carotid, is it anastomosed to the right carotid in an end-to-side configuration by using a continuous 7.0 Prolene suture (Ethicon, Cincinnati, Ohio).

Following completion of the first anastomosis, a second arteriotomy is created directly opposite the first (on the lateral segment of the
RCA). The harvested vein segment is then sutured to the lateral arteriotomy in an end-to-side configuration with 7.0 Prolene, with the distal segment of the vein closed with a permanent vascular clip to form the aneurysm fundus. Temporary clips are then removed, and any sites of bleeding from the vein pouch repaired with sutures. The height of the aneurysm vein pouch is then adjusted to create the desired size of aneurysm. The incision is closed in multiple layers over Penrose drains, which are typically left for 24 to 48 hours.

\section{Endovascular Treatment}

Endovascular treatment was performed 6 weeks or more after surgical construction of aneurysms in 13 dogs, by using coaxial microcatheters introduced by a transfemoral percutaneous approach. Balloonassisted coil embolization was performed in 3 animals, by using bare platinum coils (Micrus Endovascular, Renens, Switzerland). A first framing coil of 0.015 -inch caliber was introduced, with additional packing accomplished by using coils of decreasing diameters and calibers until they could no longer be introduced inside the aneurysm.

Stent placement was performed in 10 animals, by using balloonexpandable stents in 3 cases (Pharos stent; Micrus Endovascular, Renens, Switzerland; lengths $25-30 \mathrm{~mm}$ and diameters $3.0-4.5 \mathrm{~mm}$ ) or closed-cell self-expandable nitinol stents in 7 cases (Leo, Balt, Montmorency, France; lengths $18-25 \mathrm{~mm}$ and diameters 3.0-4.5 $\mathrm{mm})$. They were deployed from the distal $(n=4)$ or proximal $(n=4)$ RCA to the LCA, in an attempt to bridge the aneurysm neck (Fig 2 ). In 2 animals double stent placement to the 2 bifurcation branches was attempted with balloon-expandable stents or closed-cell nitinol stents. Stent diameters were chosen by estimating the caliber of the parent vessel on digital angiography, then intentionally oversized by $0.5 \mathrm{~mm}$ to promote stent stability.

\section{Ultrasonography}

All aneurysms were imaged by $4 \mathrm{MHz}$ cervical Doppler color-coded duplex sonography (linear, 7.5-12 MHz; sectoral, 5-7.5 MHz; ATL HDI 5000 U; Philips Medical Systems, Bothell, Washington) immediately after surgery, at $3.5 \pm 1.5$ weeks, and immediately before endovascular treatment. Velocities were recorded in the LCA, $1 \mathrm{~cm}$ proximal to the bifurcation (V1), in the RCA, at the level of the 2 branches of the bifurcation, $1 \mathrm{~cm}$ proximal (V2) and distal (V3) to the
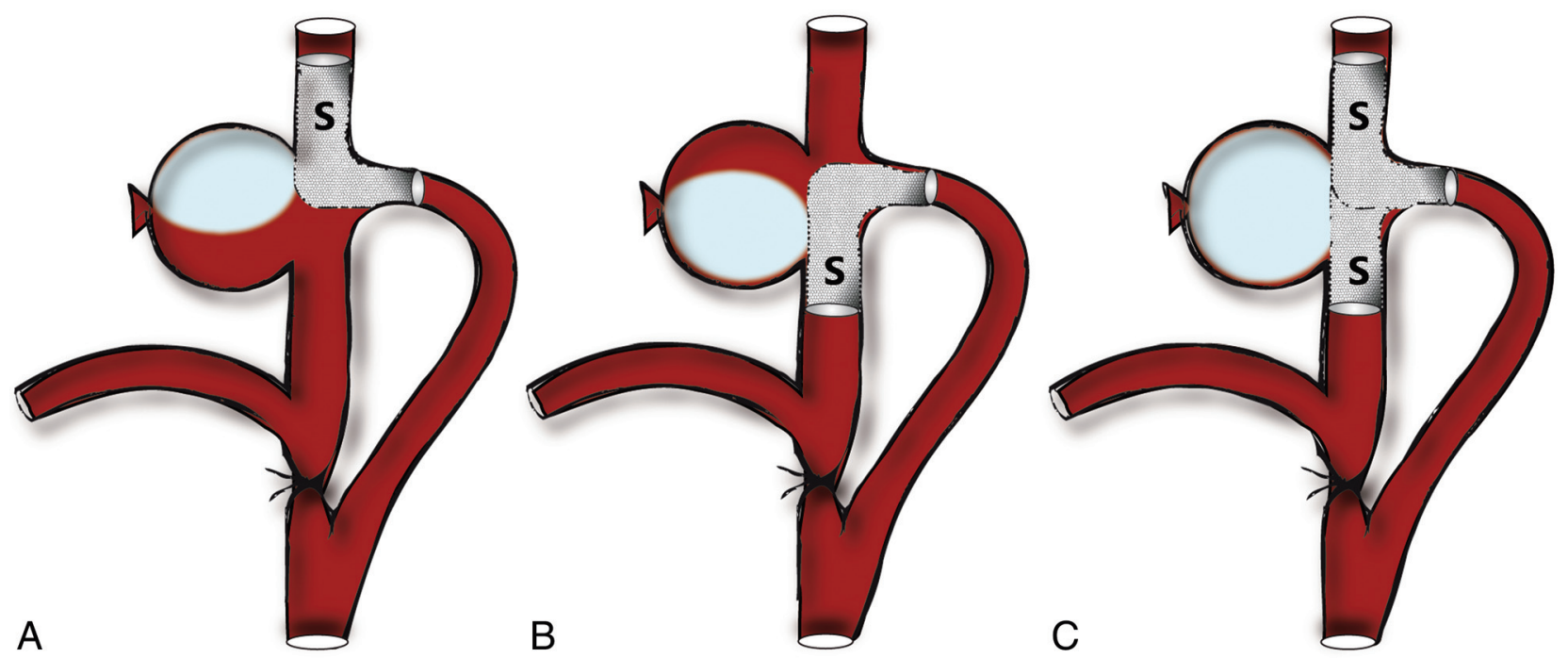

Fig 2. Stent placement. $A$, Stent $(S)$ deployed from the left carotid to distal or $(B)$ proximal RCA, bridging a portion of the aneurysm neck. $C$, Double stent technique incorporating stents in both distal and proximal RCAs. 
Table 1: Evolution of aneurysm sizes

\begin{tabular}{|c|c|c|c|}
\hline & Postsurgery & $\begin{array}{l}\text { 1-Month } \\
\text { Control }\end{array}$ & $\begin{array}{l}\text { Before Endovascular } \\
\text { Procedure } \\
(9.0 \pm 3.6 \text { months })\end{array}$ \\
\hline Fundus (mm) & $13.9 \pm 3.3$ & $18.4 \pm 5.9^{\mathrm{a}}$ & $17.2 \pm 5.6^{\mathrm{c}}$ \\
\hline Neck (mm) & $3.6 \pm 1.2$ & $4.3 \pm 0.9^{b}$ & $4.9 \pm 1.03^{c}$ \\
\hline
\end{tabular}

Note:- ${ }^{\mathrm{a}} P=.006 ;{ }^{\mathrm{b}} P=.002 ;{ }^{\mathrm{c}} P=$ nonsignificant.

anastomosis. Furthermore, we studied inflow (V4) and outflow (V5) of the aneurysm, as well as aneurysm and neck dimensions. Aneurysms were classified as occluded, partial thrombosis, no thrombus.

\section{Angiography}

Transfemoral angiography was performed $3.5 \pm 1.5$ weeks after surgery, immediately before and after endovascular procedure $(9.0 \pm 3.6$ months after surgery), and before sacrifice (11.8 \pm 3.9 months after surgery). Angiographic results were scored according to a previously described classification. ${ }^{5}$ A score of 0 indicated complete obliteration; 1 , "dog ears"; 2, residual or recurrent neck; 3 , residual aneurysm; and 4 , aneurysm unchanged or enlarged.

\section{Sacrifice and Macroscopic Photography}

Euthanasia by barbiturate overdose was performed at $11.8 \pm 3.9$ months after surgery. After a $10 \%$ formalin fixation, the carotid artery bifurcation was longitudinally opened and photographed by using a computerized imaging system (Vision PE; Clemex Technologies, Montreal, Canada). Morphologic results were graded by consensus of 2 readers. A score of 0 indicated complete occlusion; a score of 1 indicated $>50 \%$ of the orifice of the aneurysm neck covered by neointima; a score of 2 indicated $10 \%-50 \%$ coverage; a score of 3 indicated no neointima at the neck.

\section{Statistics}

We compared fundus and neck sizes after surgery, at 4 weeks, and immediately before endovascular treatment; velocities at 4 weeks and immediately before endovascular treatment by using Student $t$ tests for paired variables. A $P$ value of $<.05$ was considered significant.

\section{Results}

All animals tolerated the surgical, angiographic, and embolization procedures without complications. Surgical difficulties were encountered in 2 cases (not shown) where clipping of the innominate artery would also have occluded the subclavian and/or carotid arteries. T-type bifurcation aneurysms had a mean \pm SD fundus and neck size of $13.9 \pm 3.3 \mathrm{~mm}$ and $3.6 \pm$ $1.2 \mathrm{~mm}$, respectively, as measured by sonography, immediately after surgery.

All constructed aneurysms were angiographically patent at 4 weeks and at the end of the follow-up (mean follow-up \pm $\mathrm{SD}, 9.6 \pm 3.6$ months). Aneurysms grew in size with time, as documented at the first follow-up examination $(3.5 \pm 1.5$ weeks). Both fundus size $(18.4 \pm 5.9 \mathrm{~mm}, P=.006)$ and neck size $(4.3 \pm 0.9 \mathrm{~mm}, P=.002)$ were significantly larger. Aneurysms were stable after the initial increase in size, as documented immediately before treatment (Table 1). Both aneurysm outflow and RCA velocities distal to the aneurysm significantly decreased $(70 \pm 40$ versus $40 \pm 28 \mathrm{~cm} / \mathrm{s}, P=.003$ and $120 \pm 34$ versus $82 \pm 33 \mathrm{~cm} / \mathrm{s}, P=.03$, respectively) between 4 weeks and $9.6 \pm 3.6$ months, whereas velocities
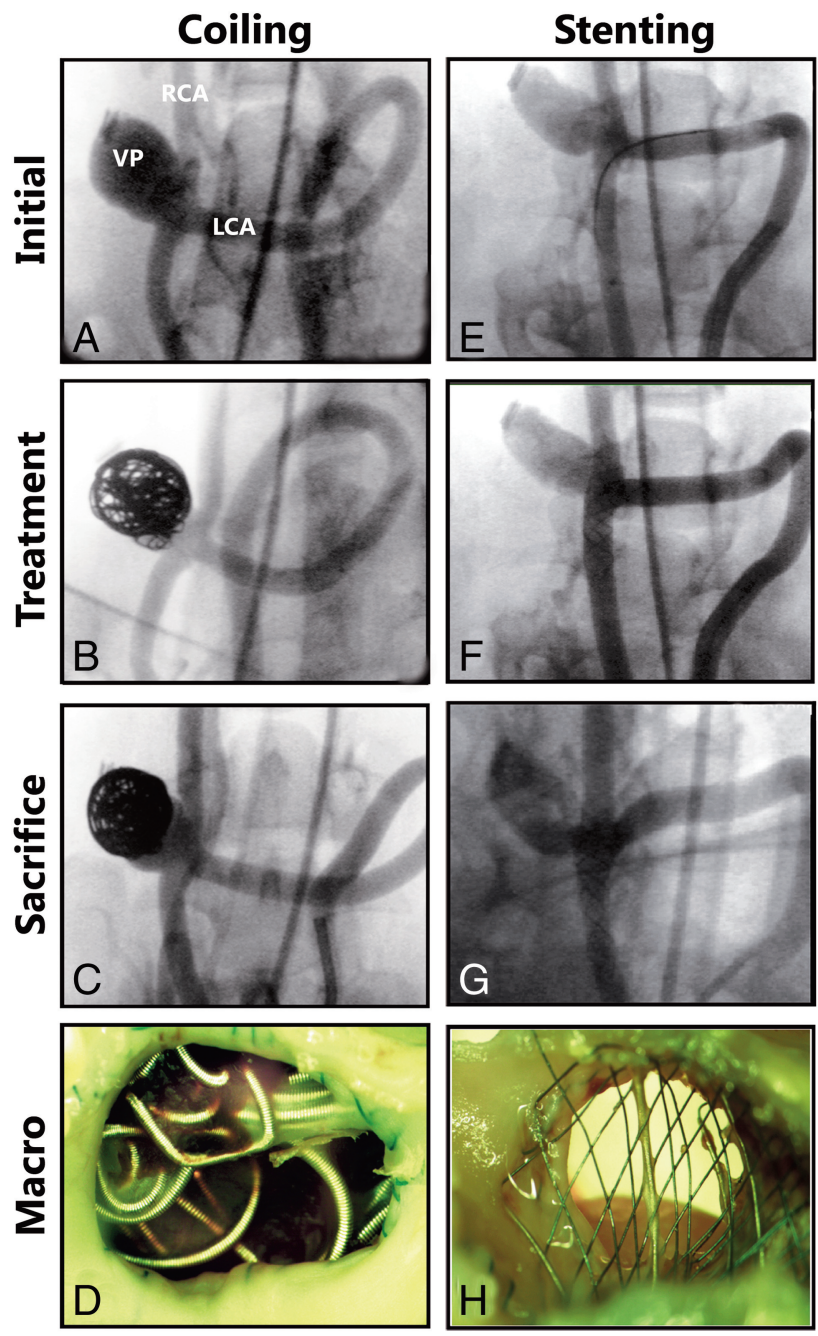

Fig 3. Angiographic results following coiling and stentings. A, Left carotid angiography performed before coiling, $(B)$ immediately following, and $(C) 10$ months after coil embolization, showing a recurrent aneurysm (score $=3$ ). $D$, Macroscopic en-face view of aneurysm ostium demonstrating the formation of some neointima around the intraaneurysm coil mass (score $=2$ ). E, Left carotid angiography with selection of the proximal RCA before, $(F)$ immediately following, and $(G) 12$ months following aneurysm treatment with a single high-porosity stent. Note the widely patent aneurysm. $H$, En-face view of aneurysm orifice covered with stent struts with negligible neointima formation on the stent.

recorded in the LCA, the RCA proximal to the aneurysm, and aneurysm inflow did not significantly change.

Aneurysm catheterization was uncomplicated. Balloon assisted-coil embolization could be performed in a fashion similar to that in clinical practice in all 3 cases. Despite satisfactory initial results, all coiled aneurysms recurred at 3 months, as shown by worse angiographic scores at follow-up and incomplete neck closure at macroscopic photography (Fig $3 A-D$ and Table 2).

Catheterization of branches for stent placement was difficult, but could be achieved in all cases, with a procedure duration of $107 \pm 25$ minutes. Technical difficulties included kinking of the stent $(n=1)$, delayed stent migration $(n=5)$, and in-stent thrombosis without occlusion of the aneurysm $(n=1)$. At 3-month follow-up, all aneurysms treated with stents were patent (Fig $3 E-H$ and Table 2). Macroscopic pathologic results were consistent with angiographic results: 1 aneurysm was class 1,1 was class 2 , and 8 were class 3 (Table 2). 


\begin{tabular}{|c|c|c|c|c|}
\hline Device & Baseline Fundus/Neck (mm) & Initial Angiographic Score & Angiographic Follow-Up Score & Macroscopic Score \\
\hline \multicolumn{5}{|l|}{ Closed-cell nitinol stent } \\
\hline 1 distal & $17.1 / 3.2$ & 4 & 3 & 2 \\
\hline 1 distal & $10.0 / 4.5$ & 4 & 4 & $3^{a}$ \\
\hline 2 distal & $17.5 / 4.1$ & 4 & 4 & $3^{\mathrm{a}}$ \\
\hline 2 distal & $11.9 / 4.1$ & 4 & 4 & $3^{a}$ \\
\hline 1 proximal & $19.2 / 2.2$ & 4 & 4 & $3^{\mathrm{a}}$ \\
\hline 2 proximal & $14.7 / 2.6$ & 4 & 3 & 3 \\
\hline 1 distal +1 proximal & $11.0 / 3.0$ & 4 & 3 & 1 \\
\hline \multicolumn{5}{|l|}{ Balloon-expandable stent } \\
\hline 1 proximal & $12.1 / 2.8$ & 4 & 4 & $3^{\mathrm{a}}$ \\
\hline 1 proximal & $8.9 / 2.1$ & 4 & 3 & 3 \\
\hline 1 distal +1 proximal & $11.1 / 2.5$ & 4 & 2 & 3 \\
\hline \multicolumn{5}{|l|}{ Coils } \\
\hline Bare platinum & $15.4 / 5.7$ & 1 & 3 & 2 \\
\hline Bare platinum & $16.8 / 5.9$ & 0 & 3 & 2 \\
\hline Bare platinum & $13.9 / 3.6$ & 0 & 2 & 2 \\
\hline
\end{tabular}

a Delayed stent migration.

\section{Discussion}

Canine carotid bifurcation aneurysms have previously been described..$^{6-9}$ Depending on the type of bifurcation, recurrence after coiling may occur with variable frequency. A T-type bifurcation model with a well-defined neck can be coiled completely, while a Y-type model, with a wide neck incorporating the origin of the LCA, cannot be completely excluded, and more frequently leads to recurrences. ${ }^{10}$ One of the difficulties encountered in deploying stents across bifurcation aneurysms arises from the angle at which the efferent blood vessels diverge from the parent vessel. In previously described bifurcation models, the vessel geometry tends to remodel after surgery and the angles between the parent artery and its branches increases, facilitating catheterization and stent placement. The $90^{\circ}$ anastomosis between the 2 carotid arteries and the natural disposition of the RCA ensures, in this new model, a $180^{\circ}$ angle between the side branches, a difficult anatomy and additional strain for stent placement. The realistic difficulties reproduced in this model may allow the evaluation of some device characteristics, as shown by the occurrence of kinking and migration of stents in this pilot project. The model was designed to test stent placement of bifurcation aneurysms, which could be accomplished from the LCA, to either 1 or both side branches, or even "transverse" stent placement of the bifurcation, which could be accomplished from the RCA before ligation of the innominate artery, or by direct puncture after ligation. Another potential use of the model could be in comparing the effects of the direction of flow on recurrences, because the bifurcation model is in effect a lateral wall aneurysm model, with a perpendicular end-to-side anastomosis. The model could be used to study various flow conditions and their effects on recurrences, on the same aneurysm construction, by omitting the innominate artery ligation (resulting in unidirectional flow toward the distal carotid artery), the left-to-right anastomosis (resulting in a lateral wall configuration), or by using both (bidirectional T-type bifurcation configuration).

Large wide-neck bifurcation aneurysms are still a challenge for complete endovascular coiling; they have a stronger propensity for residual necks and recurrences after coil embolization. ${ }^{11}$ A model designed to test the effect of new devices on recurrences after endovascular treatment must reproduce the clinical problem new devices are intended to address. ${ }^{3}$ The fact that we could show recurrences in all animals treated by coil embolization is promising, though a larger number of animals studied in a multicentric fashion may be important to validate the propensity of the model for recurrences. ${ }^{4}$

High porosity intracranial stents are increasingly being used, either to allow coiling of difficult aneurysms or to reduce the risks of recurrences. ${ }^{12,13}$ Unfortunately, their efficacy has never been proved in preclinical or clinical studies. The present model could be used to validate the claim that the use of high porosity stents in addition to coiling may decrease the risks of recurrences. The use of these stents alone, without concurrent coiling, has not been effective in the current study; a more appropriate protocol would compare coiling alone with stent placement after coiling, a study that would necessitate a larger number of animals and higher costs than what was possible in this pilot project. Different devices and various configurations (such as single or double Y-stent placement) could be compared by using this new model. Additionally, new low-porosity devices (often referred to as flow diverters) initially developed to treat large proximal aneurysms ${ }^{14}$ are being increasingly used in difficult clinical settings involving a bifurcation. They may show increased efficacy in occluding aneurysms by themselves, but the potential morbidity associated with branch occlusions is a concern that could be tested by using the same model. The model could also be found valuable to train interventionists in mastering certain technical difficulties.

The flow characteristics of our model were found with ultrasonography to be complex and unpredictable, as expected with terminal bifurcation aneurysms with balanced outflows. ${ }^{15}$ Computational fluid dynamic studies have shown extreme sensitivity of aneurysm flow patterns to parent vessel curvature and angulation, ${ }^{16,17}$ but we were unable to detect gross differences in aneurysm flow characteristics despite the minor, unavoidable anatomic differences between animals.

This preliminary study suffers several limitations, including a small number of animals, heterogeneous material, and ongoing protocol modifications. The surgical construction is no more difficult than other models, but it still necessitates skills in experimental surgery. Ligation of the innominate ar- 
tery is not always easy or possible; hence, angiographic demonstration of the anatomy is recommended before surgery.

Only 1 aneurysm can be studied per animal, a significant issue in a world of limited resources and ethical considerations for animal experimentation. The model shares with other surgical constructions some drawbacks previously mentioned in the literature, such as surgical trauma, artificial neck, and the presence of suture material. ${ }^{4}$

\section{Conclusions}

This new carotid bifurcation aneurysm model can reproduce the problem of recurrence after coiling; it could be helpful in the evaluation of new aneurysm devices such as stents and could find a role in the training of interventional specialists.

\section{Acknowledgments}

We thank Micrus Endovascular and Balt Extrusion for generously providing the stents and coils.

\section{References}

1. Molyneux A, Kerr R, Stratton I, et al. International Subarachnoid Aneurysm Trial (ISAT) of neurosurgical clipping versus endovascular coiling in 2143 patients with ruptured intracranial aneurysms: a randomised trial. Lancet 2002;360:1267-74

2. Raymond J, Guilbert F, Weill A, et al. Long-term angiographic recurrences after selective endovascular treatment of aneurysms with detachable coils. Stroke 2003;34:1398-403

3. Raymond J, Salazkin I, Gevry G, et al. Interventional neuroradiology: the role of experimental models in scientific progress. AJNR Am J Neuroradiol 2007;28:401-05

4. Bouzeghrane F, Naggara O, Kallmes D, et al. In vivo experimental aneurysm models: a systematic review. AJNR Am J Neuroradiol 2010;31:418-23 Epub 2009 Oct 29

5. Raymond J, Desfaits AC, Roy D. Fibrinogen and vascular smooth muscle cell grafts promote healing of experimental aneurysms treated by embolization. Stroke 1999;30:1657-64

6. Raymond J, Salazkin I, Metcalfe A, et al. High-concentration ethylene vinyl alcohol copolymer and endovascular treatment of experimental aneurysms: feasibility of embolization without protection devices at the neck. AJNR Am J Neuroradiol 2003;24:1778-84

7. Raymond J, Salazkin I, Georganos S, et al. Endovascular treatment of experimental wide neck aneurysms: comparison of results using coils or cyanoacrylate with the assistance of an aneurysm neck bridge device. AJNR Am J Neuroradiol 2002;23:1710-06

8. Raymond J, Berthelet F, Desfaits AC, et al. Cyanoacrylate embolization of experimental aneurysms. AJNR Am J Neuroradiol 2002;23:129-38

9. Raymond J, Salazkin I, Metcalfe A, et al. Lingual artery bifurcation aneurysms for training and evaluation of neurovascular devices. AJNR Am J Neuroradiol 2004;25:1387-90

10. Raymond J, Darsaut T, Salazkin I, et al. Mechanisms of occlusion and recanalization in canine carotid bifurcation aneurysms embolized with platinum coils: an alternative concept. AJNR Am J Neuroradiol 2008;29:745-52

11. Fernandez Zubillaga A, Guglielmi G, Vinuela F, et al. Endovascular occlusion of intracranial aneurysms with electrically detachable coils: correlation of aneurysm neck size and treatment results. AJNR Am J Neuroradiol 1994;15:815-20

12. Kis $B, W$ Weber W, Berlit $P$, et al. Elective treatment of saccular and broad-necked intracranial aneurysms using a closed-cell nitinol stent (Leo). Neurosurgery 2006;58:443-50

13. Benitez RP, Silva MT, Klem J, et al. Endovascular occlusion of wide-necked aneurysms with a new intracranial microstent (Neuroform) and detachable coils. Neurosurgery 2004;54:1359-67

14. Kallmes DF, Ding YH, Dai D, et al. A new endoluminal, flow disrupting device for treatment of saccular aneurysms. Stroke 2007;38:2346-52

15. Steiger HJ, Liepsch DW, Poll A, et al. Hemodynamic stress in terminal saccular aneurysms: a laser-Doppler study. Heart Vessels 1988;4:162-69

16. Rhee K, Han MN, Cha SH. Changes of flow characteristics by stenting in aneurysm models: influence of aneurysm geometry and stent porosity. Ann Biomed Eng 2002;30:894-904

17. Ford MD, Lee SW, Lownie SP, et al. On the effect of parent-aneurysm angle on flow patterns in basilar tip aneurysms: towards a surrogate geometric marker of intra-aneurismal hemodynamics. J Biomech 2008;41:241-48 